\title{
Evaluation of Kyo-Yuzen-Zome Fabrics with Different Pastes
}

\author{
Takashi Furukawa $^{1, *}$, Yuka Takai ${ }^{2}$, Akihiko Goto $^{2}$, Noriaki Kuwahara ${ }^{1}$, \\ and Noriyuki Kida ${ }^{1}$ \\ ${ }^{1}$ Kyoto Institute of Technology, Kyoto, Japan \\ t-furukawa@hishiken.co.jp, \\ \{nkuwahar, kida\}@kit.ac.jp \\ ${ }^{2}$ Osaka Sangyo University, Osaka, Japan \\ \{takai,gotoh\}@ise.osaka-sandai.ac.jp
}

\begin{abstract}
Yuzen-zome" is a traditional but still popular method of dyeing fabrics in Japan. The products using the Yuzen-zome method and manufactured in Kyoto city are called "Kyo-Yuzen-zome." The dyeing method of Yuzen-zome can be dividing into 10 procedures. A specialized craftsman is in charge of each procedure. During the paste application (Nori-oki) procedure, the expert applies a starch paste or a rubber paste to a fabric. The two pastes create different effect on the dyed fabric. At market, the fabric with a starch paste application is perceived to have a higher value than that with a rubber paste. In this study, the difference of the viscosity between two materials was clarified, and specimens which craftsman dyed were observed. Then how two materials put on fabrics, and the structures of them were measured.
\end{abstract}

Keywords: Paste, Fabric, Dyeing, Starch, Rubber.

\section{Introduction}

There are some methods to dye a kimono in Japan such as Yuzen-zome (Yuzen dyeing), Shibori-zome (tie dyeing), Ai-zome (indigo dyeing), and Rou-zome (batik dying). Among these methods, the most widely used dyeing technique is Yuzen-zome. The Yuzen-zome dyed in Kyoto is specifically called "Kyo-Yuzen-zome." Not much literature clarifies the origin of Yuzen-zome; however, the "Genji Hiinagata" published in 1686 indicate the names of Yuzen-zome and Yuzensai, with the descriptions of pattern dying such as Icchin-zome, Chaya-zome, and Edo-zome. Yuzensai Miyazaki is alleged to be a fan painter, Yuzensai Miyazaki, living in Kyoto at that time. Later, Kyo-Yuzen-zome has been supporting the garment industry in Japan together with Kaga-Yuzen and Edo-Yuzen. In the Meiji era, the dyeing technology of the synthetic dye was advanced, and most of the currently used dyes are synthetic dyes. Likewise in the Meiji era, once the technique to dye a fabric using a dyeing stencil was established, a mass production of kimono using Yuzen-zome became possible.

\footnotetext{
* Corresponding author.
} 
The amount of products had been increased and the annual production reached to $16,500,000$ bolts in 1971; however, it has been decreased and reached to 840,000 bolts in 2003 along with the lifestyle change [1]. Regarding the market size of Japanese dress industry, it was 627 billion yen in 2003, but was 400 billion yen in 2008, [2], and has been believed to be lower than 300 billion yen in 2013.

Generally, Kyo-Yuzen-zome can be classified into five types: hand-drawn Yuzen, kata Yuzen (i.e., dyed with paper patterns), screen printed, machine printed, and inkjet dyeing. Among them, the representative example is the hand-drawn Yuzen which whole production procedure is performed by handwork. This shows the uniqueness that each product has its own character. Each craftsman is in charge of each manufacturing procedure. In the process of producing the hand-drawn Yuzen, the outline of the design was dawn on the fabric with a dayflower extract. Then, using the paper tube, a fine line paste or "itome" is applied to the fabric to mask the line, and insert the dyes within the area with the target color. As this method masks the line of the pattern with a paste, it is also called as Itome-Yuzen.

As the flow chart shows in Figure 1, the dyeing process of hand-drawn Yuzen consists of ten procedures. The first procedure, "preliminary sketch," is to draw a pattern of the design on a white fabric with a dayflower extract. The second procedure called "paste application (i.e., masking)"is a procedure to apply a fine line paste along with the lines of the preliminary sketch to avoid the dyes to bleed other parts when inserting colors on the fabric. Figure 2 shows the scene of a paste application during the paste application procedure. The third procedure, "covering with paste," is to apply a paste on the pattern to avoid the dyes to penetrate the parts of design in brush dyeing. The fourth procedure, "brush dyeing (or hiki-zome)", is a procedure to dye evenly or gradate by a brush, using a combined colors. The fifth procedure, "steaming," is to place the brush dyed fabric in a steamer and steams it at the temperature of about $100^{\circ} \mathrm{C}$ for 20 to 50 minutes. By steaming the fabric for 20 to 50 minutes, the ground color (dye) is stabilized on the fabric. The sixth procedure, "sink yuzen, or mizumoto,"is to wash the fabric with water to eliminate the excess dyes, medicaments, and pastes from the fabric on which dyes are completely stabilized. The seventh procedure, "inserted yuzen" is a procedure to give colors to the design by using different types of brushes. The eighth procedure, "straightening the fabric or Yunoshi" is to steam wrinkles out of the fabric to soften the texture. The ninth procedure, "gold glazing" is to add here gold and silver foils and powders on the dyed fabric to make the product gorgeous. The tenth procedure, "embroidery," is to embroider a part of the design with gold and silver threads and other colory embroidery threads, to add elegance and luxuriousness to the product by giving the design volume.

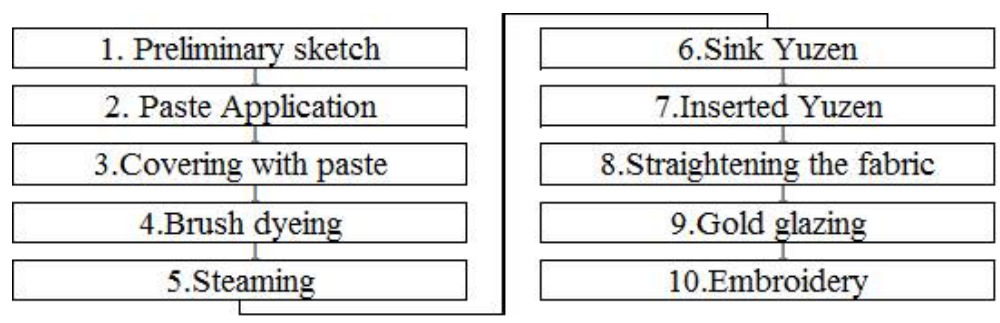

Fig. 1. Flow chart of manufacturing process 


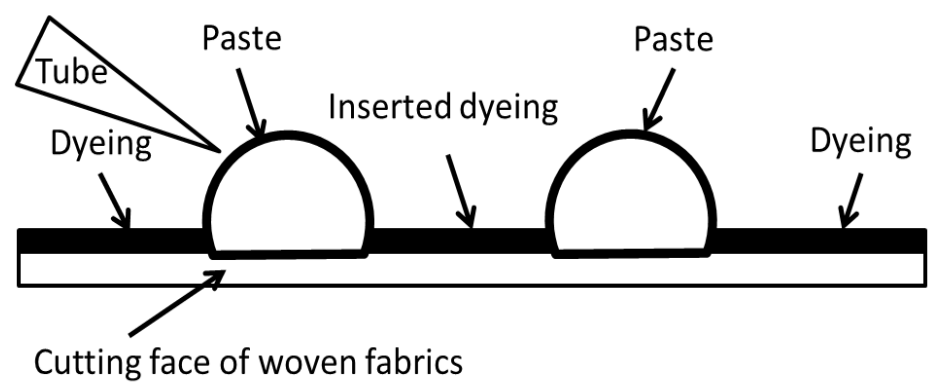

Fig. 2. Model of process about Paste application

\section{Paste Application and the Function}

\subsection{Paste Application}

In Japanese dress industry, inventing and deciding the first design to draw on kimono is considered to influence strongly on the end product. Without a high artistic quality or an aesthetic sense for the design, the product will remain unsold regardless of each procedure of making Kyo-Yuzen-zome was completed in a careful manner. The process to draw a design on a silk fabric is a preliminary sketching. The paste application procedure plays a significant role in changing highly artistic product drawn during the preliminary sketching into a manufactured product. Furthermore, recently, in many cases, instead of drawing the preliminary sketch on the silk fabric, a new method is used; a craftsman places a paper template of kimono with designs under the silk fabric and traces the design during the paste application. Therefore, it is very important to complete the putting a paste procedure by tracing the design accurately while taking the artistic quality of the designs drawn during the preliminary sketch or on the paper template.

Materials used for a paste application procedure are a starch paste, a rubber paste, and a flour. The typical materials are a starch paste and a rubber paste, which consists of over $99 \%$ of the material used in the process. There is a research on paste application and dyeing technology. In 1984, Kyoto City Senshoku Shikenjou (presently, the Kyoto Municipal Institute of Industrial Technology and Culture) published the "Technology and Techniques of Hand-Drawn Yuzen-Zome" [3], which editor-atlarge was the Committee of the Technology and Techniques of the Hand-Drawn Yuzen-Zome. While a starch paste is made from sticky rice, a rubber paste is made by melting a rubber sheet with benzin and gasoline. Currently, more than $90 \%$ of handdrawn Yuzen which were produced in Kyoto uses the rubber paste.

\subsection{Objective of Research}

On the other hand, in the Japanese dress industry, it is said that dyeing method using a starch paste during the paste application procedure makes the end products have an impression of softness and depth than using a rubber paste. Consequently, the final 
product value becomes higher when using a starch paste than a rubber paste; in many cases, the product using the starch paste tends to be sold at a higher price than the rubber paste.

In research on the relation between sensitivity evaluation and price setting, if starch paste is used as a result of evaluating the sample dyed using related [4]. Starch paste and rubber paste of the sensitivity evaluation and price setting in the Kyoto yuzen stain which uses different paste using the questionnaire technique, if high-quality increases and rubber paste is used on the other hand, it turns out that clearness increases. Furthermore, it is clear that the factor's which the starch paste is highly set up in a price rather than rubber paste, and has on a price about price setting it is a highquality feeling. On the other hand, there are the technology and technique of Tegakiyuzen dyeing which Kyoto Municipal Institute of Industrial Technology and Culture performed in 1984 also as that of Tegaki-yuzen dyeing technology and technique investigating committee editorial supervision in research on the dyeing and finishing technology of paste application or a kimono. However, researches, such as viscosity about the starch paste used for paste application and perviousness with a fiber bundle, are not made.

So, in this research, the structure dyed using starch paste and rubber paste was solved, and it aimed at clarifying relation between structure of paste application, and an impression difference and price setting. Therefore, the viscosity of starch paste and rubber paste was measured and the difference was clarified. Furthermore, by observing the section and the surface of the product produced using starch paste and rubber paste, when starch paste and rubber paste dyed, the influence which it has to a fiber bundle was investigated.

\section{Materials}

\subsection{How to Manufacture Putting Paste}

Starch paste steams and kneads rice cake rice flour and rice bran, and it mixes the solution which melted lime, paints red pigment mix it, and it needs it. Finally starch paste is filtered with the cloth of cotton. Since the antiseptic is not contained, it is easy to decompose starch paste. Therefore, it is necessary to put into a container and to save in a refrigerator. When using it again, warming in hot water must be carried out and heat must be applied. Then, rubber paste cuts board rubber finely and pickles it in benzene or gasoline together with Dan Mull liquid. Furthermore, board rubber melts by mixing the ultramarine of paints and soaking for several days, and it becomes rubber paste. The reason of red pigment mixing and mixing ultramarine with rubber paste is for using the mark color of paste at starch paste.

\subsection{Viscosity}

In the Kimono industrial world, it is said that it is hard to treat starch paste firmly compared with rubber cement. Therefore, the craftsman who can use starch paste is limited to the ability of a craftsman to use rubber cement every paste of all the members. 
Then, the viscosity of starch paste and rubber cement was measured. Production of the starch paste and rubber paste which were used for measurement was requested from the craftsman of Paste application, (70 ages, a male, right-handed person) of years-of-experience about 50 year. Indoor temperature is gone into the range of $19 \sim 20^{\circ} \mathrm{C}$, in order to carry out to viscosity measurement using a viscosity measuring device (ARES G2). The viscosity of the starch paste at the time of the shear rate 1 (/s) and rubber cement is shown in Fig. 3. As a result, it was shown that starch paste high viscosity about 30 times compared with rubber paste.

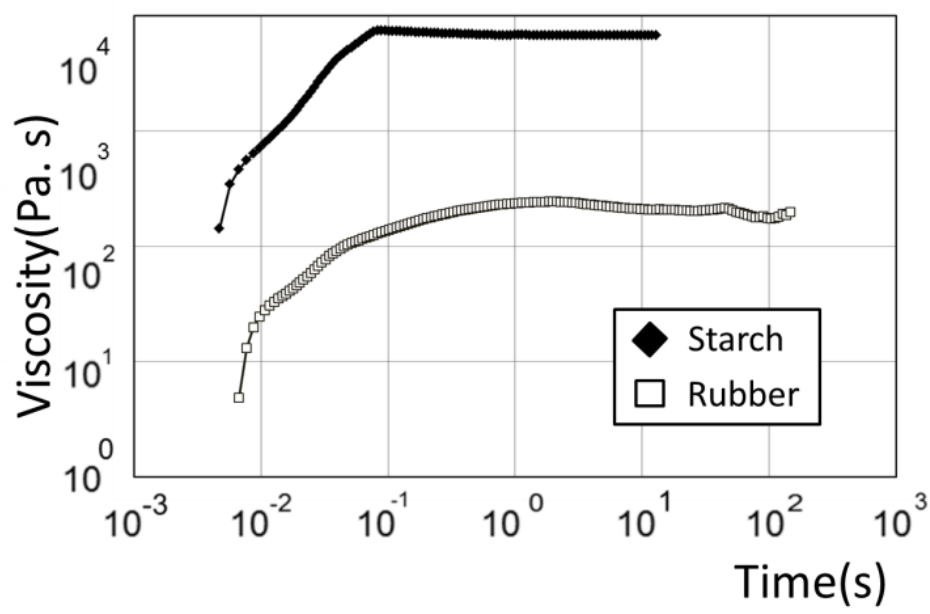

Fig. 3. Viscosity of Starch and Rubber on Shear Rate (1/s)

\section{Experiment}

\subsection{Observation of Cutting Faces}

Cloth prepared plain weave of the pure silk fabrics made from Nankyu, Inc. Manufacture was requested from the same craftsman using the same starch paste and rubber paste which were used for measurement of viscosity. The temperature which performed process of paste application was about $18^{\circ} \mathrm{C}$. The specimen performed by paste application, dyed the thing of the stage (silk has not dyed) which the ground color is not dyeing, the gradual thing which dyes a ground color and has not removed paste, and the ground color, removed two paste, and prepared the thing of the stage which became the end products. Starch and rubber judged the specimen by silk along with warp, and the observation method observed those sections. It carried out to observation using the done type metallurgical microscope (PME3: made by Olympus, Inc.) of a handstand. 


\subsection{Observation of Woven Surfaces}

In order to carry out surface observation of the part was given, a total of three things [ six ] of three sheets and rubber paste use was prepared [ the sample to which three craftsmen (more than all the members craftsman history 50 year, a male, a righthanded person) performed the process ] for the thing of starch paste use, respectively. The entire sample of six sheets chose the general pattern in the kimono industrial world called "Paddle bucket". "Paddle bucket" is a comparatively linear pattern, and it performed so that it might be parallel at warp and the woof, respectively. The size of the pattern was made into what has all six the same sheets. All were dyed through the manufacture process of the kyo-yuzen shown in Fig. 1. Each time spent on the paste application was about 30 minutes.

Cloth is pure silk fabrics and plain weave. It is a sample of these six sheets IOS D6000. A photograph was taken by make (Canon Co, Ltd). In order to suppress the variation in a light source, the indoor fluorescent light was erased and the window facing north was opened, and it adjusted so that outdoor available light might enter.

\section{$5 \quad$ Results}

\subsection{Observation of Cutting Faces}

The cross-sectional picture carried out every paste with starch paste and rubber paste is shown in the figure $4 \sim 7$. Fig. 4 shows the specimen which performs paste application and is not having the ground color dyed. Fig. 5 shows the specimen which mixed dye with starch paste and rubber paste, and performed the same process. Starch paste has blue rubber paste in reddish brown, and this is because the adhe-sion degree of paste and a fiber bundle becomes clearer. Starch paste did not permeate a fiber bundle rather than rubber paste, but adhesion area of rubber paste was larger than Fig. 4 and Fig. 5, and it turned out that it has permeated deeply into a fiber bundle. Fig. 6 shows the specimen before performing dyeing of paste application and a ground color and removing paste continuously. Adhesion area of rubber paste was larger than starch paste, and it turned out that it has permeated deeply into a fiber bundle. Fig. 7 is a sectional view of the same stage as the end products continuously. The part which removed starch paste and rubber paste was enclosed white. Starch paste did not permeate a fiber bundle deeply, but the dye which dyed the ground color has permeated the back side (back side of a dyeing side) which performed paste application. On the other hand, since rubber paste had permeated to the back side of cloth, it turned out that it has prevented a ground color entering the back side of the marks of paste. Although it was said in the kimono industry that starch paste had resist-printing power weaker than rubber paste, and tends to turn around a ground color from the reverse side of cloth, from such cross-sectional observation, rubber paste permeated the fiber bundle deeply and the resist-printing effect kept from being mixed when a ground color is dyed more became clear. 


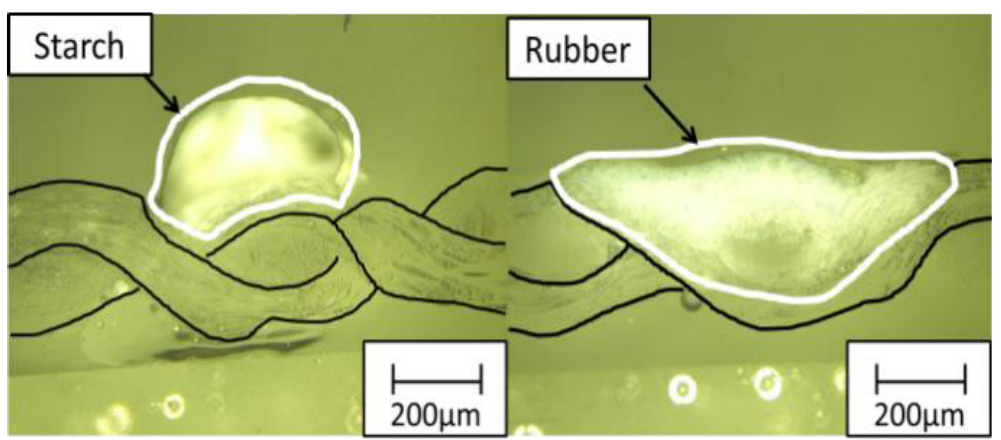

Fig. 4. Cutting faces of fabrics about Starch paste and Rubber paste before dyeing

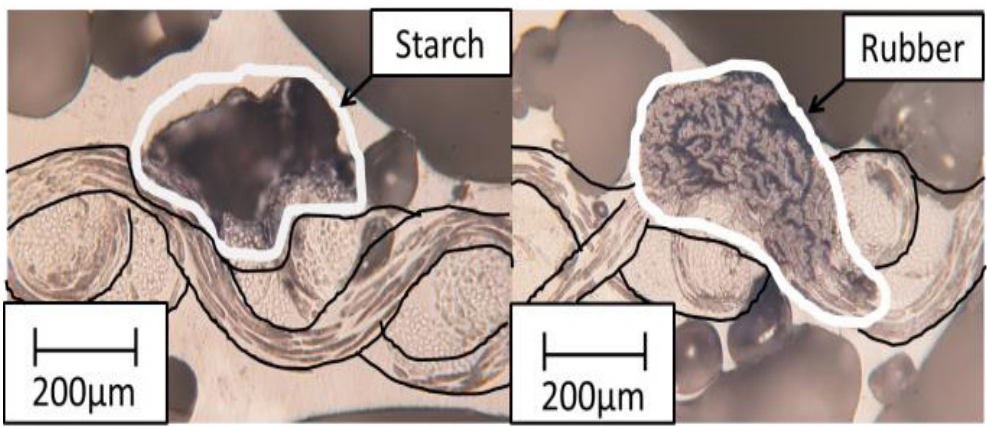

Fig. 5. Cutting faces of fabrics about Starch paste and Rubber paste mixed black dyestuff before dyeing

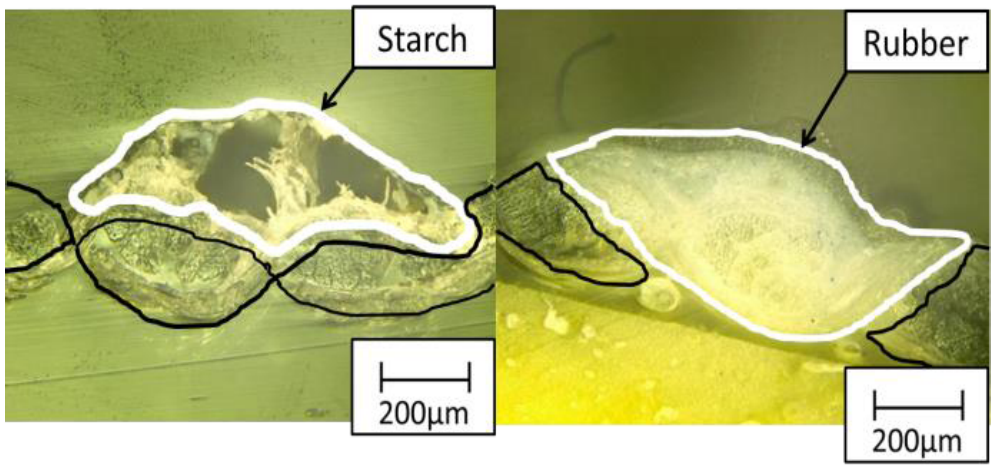

Fig. 6. Cutting faces of fabrics about Starch paste and Rubber paste before washing after dyeing 


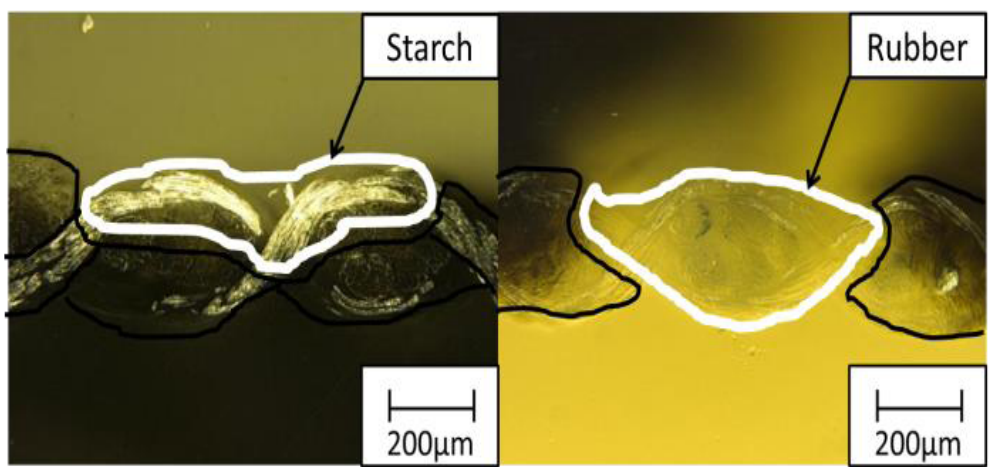

Fig. 7. Cutting faces of fabrics about Starch paste and Rubber paste after washing and steaming

\subsection{Observation of Woven Surfaces}

The sample used for surface observation is shown in Fig. 8. In the sample dyed with starch paste and rubber paste, the part which surrounded four parts enclosed with circle at a time by circle using selection and picture processing software (Photoshop, Microsoft Corp. make), respectively was cut off. Two cut-off places are perpendicular to warp, and two places which remain are parallel to warp. Four sheets were produced from the sample of one sheet. Therefore, the number of the pictures produced from surface observation is 24 . And in order to detect a RGB value from the processed picture, it outputted to the cell of Excel using matrix calculation software (MATLAB).

The average value of the RGB value of the picture which cut off the part of the starch paste 1 and rubber paste 6 are shown in Fig. 9 in the sample measured in Fig. 8. Starch paste had a change of the RGB value from a dyeing side to an achromatic side looser than rubber paste. On the other hand, the change of rubber paset from a dyeing side to an achromatic side was rapid. Furthermore, the average of the RGB value of a total of 24 places enclosed with $\mathrm{O}$ to the sample $1 \sim 6$ was computed. The sample of six sheets -- turn -- N1 (Starch), N1 (Rubber), N2 (Starch), N2 (Rubber), N3 (Starch), and N3 (Rubber) -- it named, the average value of RGB in every pixel was calculated, and those maximums were detected. Table 1 shows the average value of the maximum of the RGB value of starch paste and rubber paste about the sample of six sheets. As a result of comparing the average of the maximum of starch paste and rubber paste, the difference was seldom seen by the maximum, but the starch paste of standard deviation and a coefficient of variation are larger than rubber paste. It turned out that the maximum of a RGB value varies.

The dyed part (part by which resist printing was carried out with paste) rubber Paste to uniform being dyed firmly and the white of silk remaining firmly compared with starch paste starch paste, According to resist-printing power being weak, a ground color enters into the portion of the remains of a fine line in some places, and the white of silk does not remain completely. From such a thing, it is thought that the variation of rubber paste in an achromatic side is larger than starch paste. 


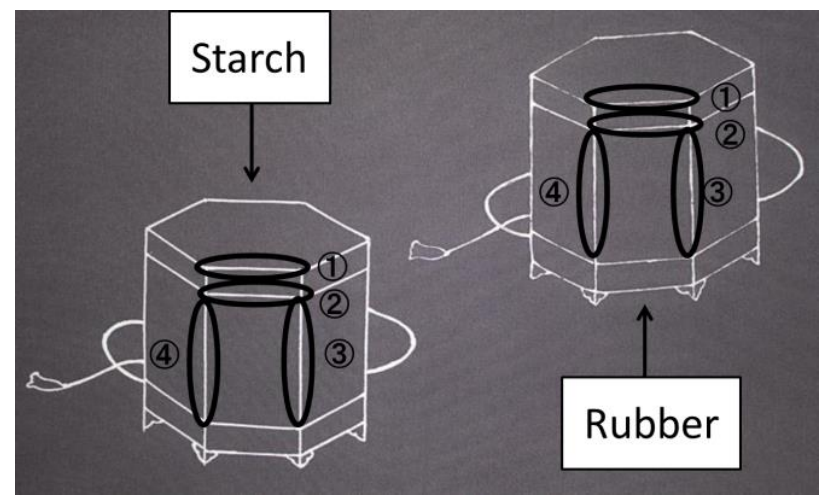

Fig. 8. Specimen for observation of woven surfaces

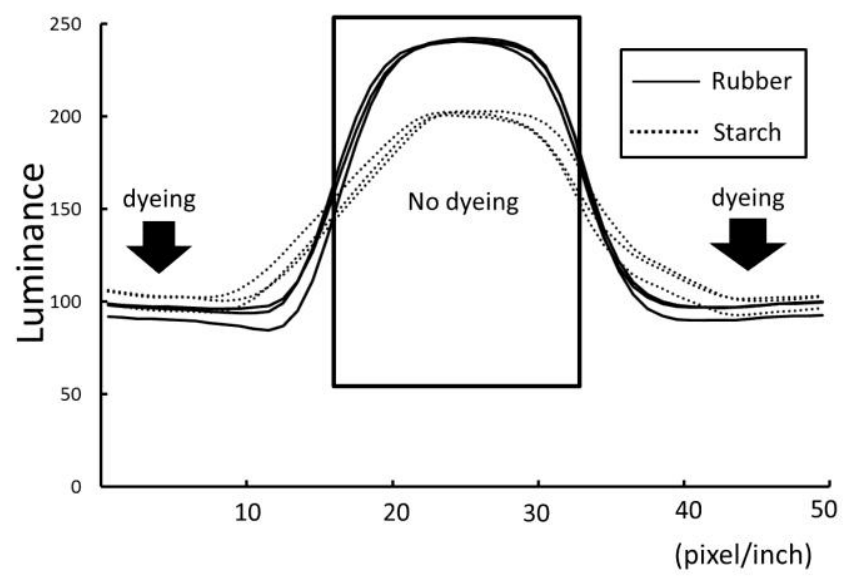

Fig. 9. Average of RGB value

Table 1. Comparison of each average

\begin{tabular}{ccccccccc} 
& \multicolumn{3}{c}{ Starch paste } & & \multicolumn{3}{c}{ Rubber paste } & material \\
\cline { 2 - 3 } & Mean & SD & CV & & Mean & SD & CV & F-value \\
\hline N1 & 234.2 & 14.4 & $6.1 \%$ & & 239.7 & 7.7 & $3.2 \%$ & $3.5 * * *$ \\
\hline N2 & 234.6 & 16.8 & $7.1 \%$ & 234.0 & 9.3 & $4.0 \%$ & $3.2 * * *$ \\
\hline N3 & 246.1 & 12.7 & $5.2 \%$ & 248.7 & 5.2 & $2.1 \%$ & $5.9 * * *$ \\
\hline
\end{tabular}




\section{Discussion}

\subsection{Cutting Face and Surface}

Two materials to a fiber bundle that rubber paste is "clear" in "superior quality" at sensitivity evaluation, and starch paste has set up price setting highly compared with rubber paste in this research how In order to investigate whether it pasted up and has affected the fiber bundle, cross-sectional observation and surface observation were performed. As shown in the figure $4 \sim 7$, starch paste has not permeated a fiber bundle compared with rubber paste. On the other hand, rubber paste has permeated the fiber bundle firmly. Although it is generally said in the kimono industrial world that the starch paste must take care and it must dye it from the reverse side of cloth by that around which dye turns (it spreads) when resist-printing power is weak and carries out Brush dyeing rather than rubber paste, it can be said that it is in agreement with understanding from this and cross-sectional observation. Furthermore, since the resistprinting effect is high compared with starch paste and rubber paste in which giving impression evaluation that starch paste is high-quality and rubber paste is clear has been reported by research of sensitivity evaluation can dye the boundary of a dyeing side and an achromatic side more vividly, it is in agreement with impression evaluation called a clear feeling. The feature of starch paste has high viscosity, resistprinting power falls compared with rubber paste, and the boundary of a dyeing side and an achromatic side does not dye vividly. Therefore, when starch paste is used from this, it is thought that it is in agreement with the result of sensitivity evaluation that a high-quality feeling can be obtained by the impression evaluation to the product of completion.

Furthermore, the sample which performed paste application by three craftsmen was prepared. The same pattern was dyed one craftsman using starch paste and rubber paste. As shown in Fig. 9, as a result of carrying out surface observation of the part in the end products, starch paste had a loose change of the RGB value compared with rubber paste. In order to investigate this result in detail, out of the sample of six sheets, four places and a total of 24 places were chosen, respectively, and surface observation was performed. As a result of calculating an average and standard deviation of each maximum, and a coefficient of variation, the difference was not seen so much by average value, but the big difference was seen by standard deviation and the coefficient of variation. Rubber paste shows from this that variation is uniformly dyed the RGB value of an achromatic side few compared with starch paste.

That is, when rubber paste is used, it is shown that the boundary of a dyeing side and an achromatic side is uniform, and it is shown that rubber paste has the feature which it can be uniform and can be dyed vividly. On the other hand, starch paste has large variation to the maximum of a RGB value, and the boundary of a dyeing side and an achromatic side does not dye vividly, but it can be said that this result is highquality and relevance with the result which can set up a price highly is suggested.

\subsection{Dyeing Model}

The result of having performed cross-sectional observation and surface observation according to material is made into a mimetic diagram, and is shown in Fig. 10. 
Cross-sectional observation and surface observation to starch paste does not permeate a fiber bundle compared with rubber paste, and its boundary of a dyeing side and an achromatic side is not clear. On the other hand, the resist-printing effect of rubber paste is high, it is uniform and the marks of paste application remain vividly. It is thought that there will be relevance with the result that starch paste is high-quality and rubber paste is clear, from the feature of starch paste and rubber paste.

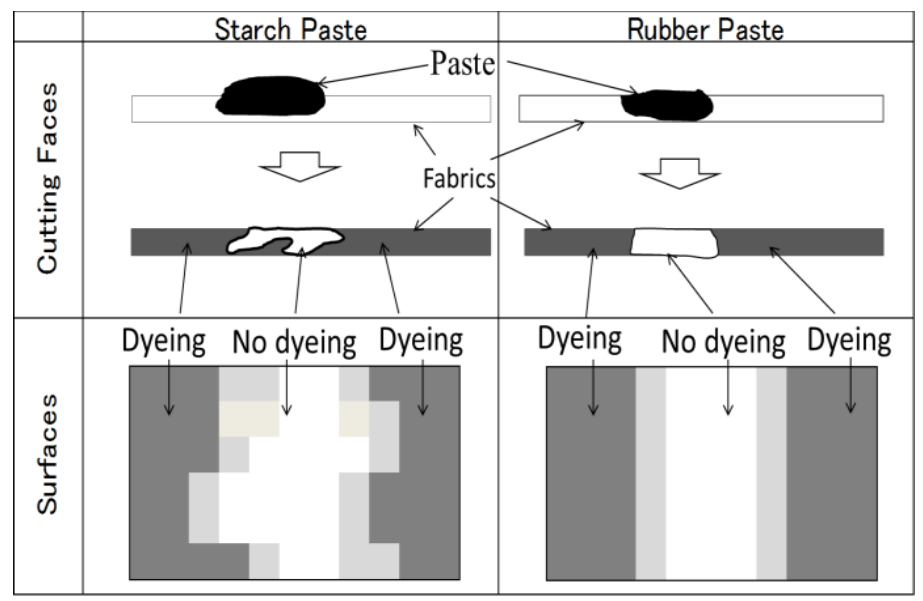

Fig. 10. Relationship between fabrics, pastes and dyeing

\section{Conclusion}

In this research, it became clear that starch paste has viscosity about 30 times as high as rubber paste. From cross-sectional observation, starch paste was understood that the resist-printing effect is which has little osmosis in a fiber bundle lower than rubber paste. Moreover, as for the starch paste, the boundary of a dyeing side and an achromatic side varies, and it became clear from surface observation that the boundary of rubber paste of a dyeing side and an achromatic side is uniform and that it is clear. Since the number of the samples produced for surface observation is six, I would like to examine whether a sample is increased and the same result is brought by this research from now on. Moreover, although the part perpendicular to warp and parallel was chosen, I would like to also perform observation of a bias part to warp.

Furthermore, comparison with the way a model performs paste application of a fine line type, and the dyeing method in which mass-production called model Yuzen other than Tegaki-yuzen is possible is also required. Moreover, although it is possible in the kimono industrial world to use it if rubber paste has a skillful degree for about one year, in order to master starch paste, it is said that it generally takes for about ten years. Furthermore, although the craftsman who can use starch paste can also use rubber paste, the craftsman only treating rubber paste cannot use starch paste. 
That is, since the number of craftsmen which can use starch paste is limited, there is very little production with starch paste than rubber paste, and the starch paste of the ratio which uses starch paste in the number of production in Tegaki-Yuzen is about $10 \%$. Then, although training of the craftsman who can use starch paste is a future subject, I would like to make low the development of rubber paste and the viscosity of starch paste which have a high-quality feeling like starch paste, and to develop the starch paste which is easy to treat also to craftsmen who can treat only rubber paste.

\section{References}

1. Kyo-yuzen Kyodoukumiai Rengoukai, Research of production in Kyo-Yuzen and KyoKomon (2012)

2. Yano Keizai Kenkyujo, Kimono Sangyo Hakusho (2009)

3. Research Committee of Technique and Skill in Tegaki Yuzen-zome, Technique and Skill in Tegaki Yuzen-zome Kyoto Municipal Institute of Industrial Technology and Culture, pp.67-100 (1984).

4. Furukawa, T.: Relationship between Kansei Evaluation and pricing Decision to Kyo-YuzenZome with Different Pastes, Japan Socuety of Kansei Engineering (2013) 